\title{
Serum albumin level as a predictor of contrast-induced acute kidney injury following coronary angiography
}

Jayakumar Sreenivasan¹, Muhammad S. Khan², Qiyu Wang², Sagar Ranka³, Tauseef Akhtar2, Jishanth Mattumpuram², Rohit Agrawal², Min Zhuo ${ }^{4}$, Wilbert S. Aronow ${ }^{1}$, Neha Yadav ${ }^{2}$

${ }^{1}$ Department of Cardiology, Westchester Medical Center, Valhalla, USA

${ }^{2}$ Department of Medicine, John H. Stroger Jr. Hospital of Cook County, Chicago, USA

${ }^{3}$ Department of Cardiology, Kansas University Medical Center, KC, USA

${ }^{4}$ Department of Nephrology, Beth Israel Deaconess Medical Center, Boston, MA

Submitted: 25 April 2020

Accepted: 27 April 2020

Arch Med Sci Civil Dis 2020; 5: e29-e34

DOI: https://doi.org/10.5114/amscd.2020.95224

Copyright (c) 2020 Termedia \& Banach

\section{Abstract}

Introduction: The association between serum albumin level and the risk of contrast-induced acute kidney injury (CI-AKI) after coronary angiography has not been well established.

Material and methods: A retrospective chart review was performed for all patients who underwent coronary angiography with or without percutaneous coronary intervention $(\mathrm{PCl})$ at a tertiary care medical center over a period of 5 years. Cl-AKI was defined as an absolute increase in creatinine of 0.3 $\mathrm{mg} / \mathrm{dl}$ from baseline within $48 \mathrm{~h}$ of contrast exposure. Mean pre-procedural albumin level was compared between patients who developed CI-AKI and those who did not. The optimal cut-off point was obtained from the receiver operating characteristic (ROC) curve and univariate multiple logistic regression analysis was used to assess the associated risk of $\mathrm{Cl}$-AKI.

Results: Among 1319 patients (females $=32.3 \%$, mean age: $58.2 \pm 13.6$ years) included in the study, 201 (15.2\%) developed CI-AKI. Baseline serum albumin (3.5 vs. $3.8 \mathrm{gm} / \mathrm{dl}, p<0.001$ ) was significantly lower in patients who developed $\mathrm{CI}$-AKI. ROC curve analysis revealed an optimal cutoff value for serum albumin of $3.85 \mathrm{gm} / \mathrm{dl}$ to predict $\mathrm{Cl}$-AKI with $67.2 \%$ sensitivity and $52.2 \%$ specificity (area under the curve $(A U C)=0.62, p<0.001$ ). Serum albumin $<3.85 \mathrm{gm} / \mathrm{dl}$ is significantly associated with higher risk of developing $\mathrm{Cl}-\mathrm{AKI}$ (adjusted odds ratio $(\mathrm{AOR})=1.5,95 \% \mathrm{Cl}: 1.1-2.1, p=0.02$ ).

Conclusions: Serum albumin $<3.85 \mathrm{gm} / \mathrm{dl}$ is an independent predictor of $\mathrm{Cl}-\mathrm{AKI}$ in patients undergoing coronary angiography.

Key words: contrast media, kidney diseases, percutaneous coronary intervention, hypoalbuminemia, coronary angiography.

\section{Introduction}

Percutaneous coronary intervention $(\mathrm{PCl})$ is the preferred treatment modality for acute coronary syndromes and progressive symptomatic coronary artery disease refractory to medical management across all age groups [1-3]. Contrast-induced acute kidney injury (CI-AKI) is one of the common complications of $\mathrm{PCl}$ [4]. Pathophysiology of $\mathrm{Cl}-\mathrm{AKI}$ is poorly understood [5]. Animal models have shown a pattern of acute tubular necrosis among patients with $\mathrm{Cl}-\mathrm{AKI}$; however, the mechanism of tubular damage is unclear $[5,6]$. Contrast causing direct chemical damage to

\author{
Corresponding author: \\ Jayakumar Sreenivasan \\ Department of Cardiology \\ Westchester Medical Center \\ United States \\ E-mail: jayakumars101@ \\ gmail.com
}


the tubular cells, contrast-induced local inflammation and cellular damage, and contrast-induced vasoconstriction causing renal tissue hypoxia are a few of the suggested mechanisms of $\mathrm{Cl}-\mathrm{AKI}$ [5, 6]. There are emerging data to suggest that $\mathrm{Cl}$ AKI may be of less clinical significance in patients undergoing contrast computed tomography (CT) studies [7]. However, $\mathrm{Cl}-\mathrm{AKI}$ remains a significant cause of morbidity and mortality in patients undergoing coronary angiography with or without coronary intervention [8]. The development of severe acute kidney injury (AKI) after contrast exposure is associated with short-term complications including the requirement for urgent renal replacement therapy, which has a high in-hospital mortality as well as worse long-term renal outcomes $[8,9]$. In 2004, Mehran et al. proposed a risk score for prediction of $\mathrm{Cl}-\mathrm{AKI}$ after $\mathrm{PCl}$, which includes hypotension, intra-aortic balloon pump (IABP) use, congestive heart failure, age $>75$, anemia, diabetes and baseline renal function [10, 11]. Most of these universal risk factors of $\mathrm{Cl}-\mathrm{AKI}$ are largely non-modifiable and irreversible. Therefore, it is important to recognize the association of some novel risk factors such as hypoalbuminemia with the development of $\mathrm{Cl}-\mathrm{AKI}$, which can potentially be modified to reduce the incidence and sequelae of $\mathrm{Cl}-\mathrm{AKI}$. Serum and urine albumin levels have been evaluated as potential markers of poor prognosis and predictors of various inflammatory diseases $[12,13]$.

In our study, we aimed to evaluate the association of serum albumin level with the incidence of $\mathrm{Cl}$-AKI in patients undergoing coronary angiography.

\section{Material and methods}

\section{Data source}

We conducted an extensive retrospective electronic chart review of all patients who underwent coronary angiography with or without percutaneous coronary intervention $(\mathrm{PCl})$ at John H. Stroger Jr. Hospital of Cook County, Chicago, USA between January 2012 and December 2016. Patients with end stage renal disease (ESRD) at the time of the procedure and those who did not have a pre-procedural serum albumin level (defined as the serum albumin level within a month prior to the procedure) were excluded. A total of 1319 patients were included in the study. Clinical and laboratory characteristics were obtained by chart review. This study was approved by John $\mathrm{H}$. Stroger Jr. Hospital of Cook County institutional research board (IRB) (Approval License Number CCH IRB ID: 17-606). IRB waived the requirement for informed consent due to the retrospective chart review study design.

\section{Definitions}

The Acute Kidney Injury Network (AKIN) criteria for $A K I$, as defined as $\geq 0.3 \mathrm{mg} / \mathrm{dl}$ rise in creatinine from baseline within $48 \mathrm{~h}$ of exposure to a nephrotoxic agent was used as the definition for $\mathrm{Cl}$-AKI in our study [14]. It is acknowledged that there is no consistent definition of $\mathrm{Cl}-\mathrm{AKI}$ among epidemiological studies. There is also no universal definition of hypoalbuminemia, and in our study, we did not use any pre-defined serum albumin level as a classification criterion. Instead, the optimal cutoff value for serum albumin level was obtained from receiver operating characteristic (ROC) curve analysis.

\section{Statistical analysis}

Mean pre-procedural albumin level was compared between $\mathrm{Cl}-\mathrm{AKI}$ and non-Cl-AKI groups by using the independent sample $t$-test. ROC curve analysis was performed to obtain the optimal cutoff point for maximal sensitivity and specificity. Area under the curve (AUC) was estimated to define the discriminatory power of the optimal cutoff value of the serum albumin level. AUC of 0.5 indicates no discriminatory power for the test under consideration and AUC closer to or more than 0.7 is considered as good discriminatory power for any test. Patients were then further classified into a low serum albumin group and a normal serum albumin group using this cut-off value for serum albumin level. Baseline demographic characteristics were compared using the independent sample $t$-test for continuous variables, and the Pearson $\chi^{2}$ test for categorical variables. Univariate multiple logistic regression analysis was used to assess the risk of $\mathrm{Cl}-\mathrm{AKI}$ associated with age, previous diagnosis of heart failure, access type (radial vs. femoral), pre-procedural hemoglobin level, cardiogenic shock and baseline chronic kidney disease. All $p$-values are 2-sided and a $p$-value $<0.05$ was considered statistically significant. Statistical analysis was performed using SPSS software (SPSS Inc., Chicago, IL).

\section{Results}

Among the 1319 patients (females $=32.3 \%$, mean age: $58.2 \pm 13.6$ years) who were included in our study, 201 (15.2\%) developed CI-AKI. Baseline serum albumin (3.5 vs. $3.8 \mathrm{gm} / \mathrm{dl}, p<0.001$ ) was significantly lower in the $\mathrm{Cl}$-AKI group. As shown in Table I, which includes all baseline characteristics and outcomes of the study population, patients with a serum albumin level < $3.85 \mathrm{gm} / \mathrm{dl}$ had a higher incidence of $\mathrm{Cl}$-AKI (20.2\%) compared to the group with a normal serum albumin level $(10.1 \%)(p<0.001)$. There was a higher proportion of African Americans and congestive heart failure 
Table I. Baseline characteristics and outcomes of study population

\begin{tabular}{|c|c|c|c|c|c|}
\hline \multirow{2}{*}{$\begin{array}{l}\text { Baseline characteristics } \\
\text { and outcomes }\end{array}$} & \multirow[t]{2}{*}{ Subgroups } & \multirow{2}{*}{$\begin{array}{l}\text { Total } \\
(n=1319)\end{array}$} & \multicolumn{2}{|c|}{ Hypoalbuminemia } & \multirow[t]{2}{*}{$P$-value } \\
\hline & & & No $(n=668)$ & Yes $(n=651)$ & \\
\hline Mean age [years] & & $58.2 \pm 13.6$ & $58.1 \pm 15.7$ & $58.3 \pm 11.1$ & 0.76 \\
\hline Female & & $425(32.2 \%)$ & $191(29.3 \%)$ & $234(35.0 \%)$ & 0.02 \\
\hline \multirow[t]{4}{*}{ Race/ethnicity: } & African American & $613(46.5 \%)$ & $268(41.2 \%)$ & $345(51.6 \%)$ & \multirow[t]{4}{*}{$<0.001$} \\
\hline & Caucasian & $279(21.2 \%)$ & $164(25.2 \%)$ & $115(17.2 \%)$ & \\
\hline & Hispanic & $229(17.4 \%)$ & $123(18.9 \%)$ & $106(15.9 \%)$ & \\
\hline & Asian & $156(11.8 \%)$ & $73(11.2 \%)$ & $83(12.4 \%)$ & \\
\hline Current smoking & & $546(41.4 \%)$ & $281(43.2 \%)$ & $265(39.7 \%)$ & 0.11 \\
\hline Hypertension & & $983(74.5 \%)$ & $510(78.3 \%)$ & $473(70.8 \%)$ & $<0.001$ \\
\hline Diabetes mellitus & & $472(35.8 \%)$ & $222(34.1 \%)$ & $250(37.4 \%)$ & 0.11 \\
\hline Prior $C A D$, stroke or $C A B G$ & & 399 (30.3\%) & $211(30.4 \%)$ & $188(28.1 \%)$ & 0.14 \\
\hline Prior heart failure & & $294(22.3 \%)$ & $127(19.5 \%)$ & $167(25.0 \%)$ & 0.01 \\
\hline Mean LVEF (\%) & & $48.7 \pm 17.1$ & $50.7 \pm 16.2$ & $46.8 \pm 17.8$ & $<0.001$ \\
\hline Statin use & & $561(42.5 \%)$ & $276(42.4 \%)$ & $285(42.7 \%)$ & 0.48 \\
\hline \multirow[t]{5}{*}{ Prior CKD: } & No CKD & $581(44.0 \%)$ & $289(44.4 \%)$ & $292(43.7 \%)$ & \multirow[t]{5}{*}{$<0.001$} \\
\hline & Stage 2 (GFR 61 to 90) & $519(39.3 \%)$ & $276(42.4 \%)$ & $243(36.4 \%)$ & \\
\hline & Stage 3 (GFR 31 to 60) & $166(12.6 \%)$ & 75 (11.5\%) & $91(13.6 \%)$ & \\
\hline & Stage 4 (GFR 15 to 30 ) & $42(3.2 \%)$ & $7(1.1 \%)$ & $35(5.2 \%)$ & \\
\hline & Stage 5 (GFR less than 15) & $11(0.8 \%)$ & $4(0.6 \%)$ & $7(1.0 \%)$ & \\
\hline \multirow[t]{5}{*}{ Clinical presentation: } & Stable angina & $186(14.1 \%)$ & $109(16.7 \%)$ & $77(11.5 \%)$ & \multirow[t]{5}{*}{$<0.001$} \\
\hline & Unstable angina & $332(24.4 \%)$ & $175(26.9 \%)$ & $147(22.0 \%)$ & \\
\hline & Non-STEMI & $429(32.5 \%)$ & $216(33.2 \%)$ & $213(31.9 \%)$ & \\
\hline & STEMI & $140(10.6 \%)$ & $57(8.8 \%)$ & $83(12.4 \%)$ & \\
\hline & Heart failure & $219(16.6 \%)$ & $83(12.7 \%)$ & $136(20.4 \%)$ & \\
\hline $\begin{array}{l}\text { Cardiogenic shock at } \\
\text { presentation or within } 24 \mathrm{~h}\end{array}$ & & $40(3.0 \%)$ & $15(2.3 \%)$ & $25(3.7 \%)$ & 0.08 \\
\hline Intra-aortic balloon pump use & & $18(1.4 \%)$ & $3(0.5 \%)$ & $15(2.2 \%)$ & $<0.001$ \\
\hline $\begin{array}{l}\text { No percutaneous coronary } \\
\text { intervention }\end{array}$ & & $902(68.4 \%)$ & $433(66.5 \%)$ & $469(70.2 \%)$ & 0.46 \\
\hline \multirow[t]{4}{*}{ No. of coronary stents: } & 1 & $309(23.4 \%)$ & $165(25.3 \%)$ & $144(21.6 \%)$ & \multirow[t]{4}{*}{0.46} \\
\hline & 2 & $90(6.8 \%)$ & $45(6.9 \%)$ & $45(6.7 \%)$ & \\
\hline & 3 & $15(1.1 \%)$ & $6(0.9 \%)$ & $9(1.3 \%)$ & \\
\hline & 4 & $3(0.2 \%)$ & $2(0.3 \%)$ & $1(0.1 \%)$ & \\
\hline Mean contrast volume [ml] & & $125.4 \pm 86.8$ & $130.6 \pm 90.3$ & $120.3 \pm 82.9$ & 0.07 \\
\hline \multirow[t]{5}{*}{ Contrast volume use [ml] } & $<100$ & $698(52.9 \%)$ & $327(50.2 \%)$ & $371(55.5 \%)$ & \multirow[t]{5}{*}{0.30} \\
\hline & $101-200$ & $404(30.6 \%)$ & $210(32.3 \%)$ & $194(29.0 \%)$ & \\
\hline & $201-300$ & $169(12.8 \%)$ & $86(13.2 \%)$ & $83(12.4 \%)$ & \\
\hline & $301-400$ & $34(2.6 \%)$ & $19(2.9 \%)$ & $15(2.2 \%)$ & \\
\hline & $>400$ & $14(1.1 \%)$ & $9(1.4 \%)$ & $5(0.7 \%)$ & \\
\hline Mean baseline $\mathrm{Hb}$ [gm/dl] & & $13.1 \pm 2.0$ & $13.5 \pm 1.9$ & $12.6 \pm 2.0$ & $<0.001$ \\
\hline
\end{tabular}


Table I. Cont.

\begin{tabular}{|c|c|c|c|c|c|}
\hline \multirow{2}{*}{$\begin{array}{l}\text { Baseline characteristics } \\
\text { and outcomes }\end{array}$} & \multirow[t]{2}{*}{ Subgroups } & \multirow{2}{*}{$\begin{array}{c}\text { Total } \\
(n=1319)\end{array}$} & \multicolumn{2}{|c|}{ Hypoalbuminemia } & \multirow[t]{2}{*}{$P$-value } \\
\hline & & & No $(n=668)$ & Yes $(n=651)$ & \\
\hline Mean GFR [ml/h] & & $86.5 \pm 31.6$ & $87.7 \pm 27.8$ & $85.2 \pm 35.0$ & 0.14 \\
\hline $\begin{array}{l}\text { Mean pre-procedural } \\
\text { creatinine }[\mathrm{mg} / \mathrm{dl}]\end{array}$ & & $1.1 \pm 0.6$ & $1.0 \pm 0.5$ & $1.1 \pm 0.7$ & $<0.001$ \\
\hline Acute kidney injury & & $201(15.2 \%)$ & $66(10.1 \%)$ & $135(20.2)$ & $<0.001$ \\
\hline $\begin{array}{l}\text { Requirement for new } \\
\text { hemodialysis }\end{array}$ & & $12(0.9 \%)$ & $1(0.2 \%)$ & $11(1.6 \%)$ & 0.03 \\
\hline $\begin{array}{l}\text { Requirement for blood } \\
\text { transfusion }\end{array}$ & & $24(1.8 \%)$ & $8(1.2 \%)$ & $16(2.4 \%)$ & 0.08 \\
\hline Length of hospital stay & & $5.2 \pm 5.1$ & $4.6 \pm 4.4$ & $5.7 \pm 5.6$ & $<0.001$ \\
\hline
\end{tabular}

$C A B G$ - coronary artery bypass grafting, CAD - coronary artery disease, CKD - chronic kidney disease, GFR - glomerular filtration rate, LVEF - left ventricular ejection fraction, STEMI - ST elevation myocardial infarction.

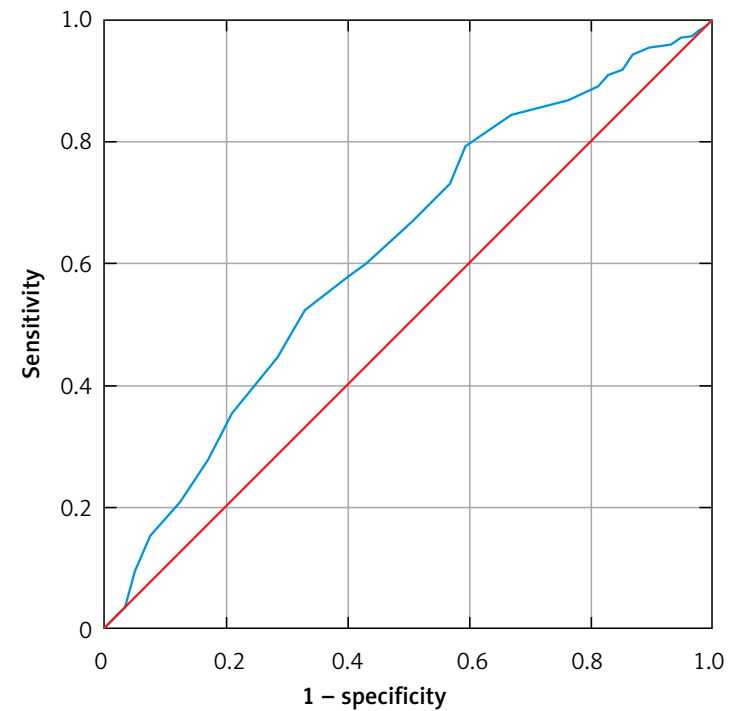

Figure 1. Receiver operating curve analysis. Receiver operating curve analysis of pre-procedural serum albumin for predicting contrast-induced acute kidney injury

Diagonal segments are produced by ties. patients in the low serum albumin group. Mean baseline hemoglobin level was lower in the low serum albumin group, and this group had a higher composition of patients with at least stage 3 chronic kidney disease (CKD), defined as estimated glomerular filtration rate (eGFR) $<60 \mathrm{ml} / \mathrm{min}$. Length of hospital stay was significantly longer in the low serum albumin group. The ROC curve analysis (Figure 1) revealed an optimal cut-off value for serum albumin as $3.85 \mathrm{gm} / \mathrm{dl}$ to predict Cl-AKI with $67.2 \%$ sensitivity and $52.2 \%$ specificity (AUC $=0.62,95 \%$ confidence interval $(\mathrm{Cl}): 0.58-$ $0.66, p<0.001)$.

In addition, serum albumin level $<3.25 \mathrm{gm} /$ dl offers a reasonably good specificity of $87.9 \%$ at the expense of poor sensitivity with a good positive predictive value in diagnosing $\mathrm{Cl}-\mathrm{AKI}$. As shown in Table II, age, radial vs femoral access, prior heart failure, intra-aortic balloon pump (IABP) use, cardiogenic shock at presentation, pre-procedural hemoglobin level, pre-existing CKD and serum albumin $<3.85 \mathrm{gm} / \mathrm{dl}$ were identified as significant predictors of $\mathrm{Cl}$-AKI following coronary

Table II. Univariate multiple regression analysis of hypoalbuminemia as an independent predictor of Cl-AKI adjusted for covariates

\begin{tabular}{|lcccc|}
\hline Variables & Odds ratio $(95 \% \mathrm{Cl})$ & $\boldsymbol{P}$-value & $\begin{array}{c}\text { Adjusted odds ratio } \\
(95 \% \mathrm{Cl})\end{array}$ & $P$-value \\
\hline Age & $1.2(1.1-1.3)$ & 0.02 & $1.0(0.9-1.1)$ & 0.32 \\
\hline Radial vs. femoral access & $2.0(1.4-2.8)$ & $<0.001$ & $2.4(0.6-5.7)$ & 0.09 \\
\hline Prior heart failure & $2.1(1.5-2.8)$ & $<0.001$ & $1.6(1.2-2.3)$ & 0.01 \\
\hline Cardiogenic shock & $2.8(1.4-5.5)$ & $<0.001$ & $1.3(0.6-3.0)$ & 0.44 \\
\hline Intra-aortic balloon pump use & $3.6(1.4-9.4)$ & $<0.001$ & $1.8(0.6-5.7)$ & 0.25 \\
\hline Pre-procedural hemoglobin & $0.6(0.5-0.7)$ & $<0.001$ & $0.7(0.6-0.8)$ & 0.00 \\
\hline Glomerular filtration rate $<60 \mathrm{ml} /$ min & $3.3(2.2-5.1)$ & $<0.001$ & $1.7(1.2-2.1)$ & 0.02 \\
\hline Serum albumin $<3.85$ gm/dl & $2.2(1.6-3.0)$ & $<0.001$ & $1.5(1.1-2.1)$ & 0.02 \\
\hline Cl-confidence interval Cl-AKI-contrast-induced acute kidney injury & & &
\end{tabular}

$\mathrm{Cl}$ - confidence interval, $\mathrm{Cl}$-AKI - contrast-induced acute kidney injury. 
angiography. Serum albumin level < $3.85 \mathrm{gm} / \mathrm{dl}$ was independently associated with higher risk of developing $\mathrm{Cl}-\mathrm{AKI}$ following coronary angiography (adjusted odds ratio $(\mathrm{AOR})=1.5,95 \% \mathrm{Cl}: 1.1-2.1$, $p=0.02)$ adjusted for all other significant covariates (Table II).

\section{Discussion}

In our study, we found that hypoalbuminemia defined as serum albumin $<3.85 \mathrm{gm} / \mathrm{dl}$ is as an independent risk factor for development of $\mathrm{Cl}$-AKI in patients undergoing coronary angiography with or without $\mathrm{PCl}$. To the best of our knowledge this is the first study in the United States to evaluate hypoalbuminemia as an important predictor of $\mathrm{Cl}$ AKI following contrast exposure. The result of our study supports the hypothesis that low albumin state serves as an independent risk factor for $\mathrm{Cl}$ AKI. It is also consistent with a previous Turkish study which identified an inverse association between serum albumin level and the development of contrast-induced AKI after urgent coronary angiography with $\mathrm{PCl}$ for patients with acute coronary syndrome [15].

Although the mechanism of $\mathrm{Cl}-\mathrm{AKI}$ is not completely understood, there are several mainstream hypotheses on how contrast agents cause renal injury: intense vasoconstriction induced by contrast media mediated by endothelin and other vasoactive substances, release of reactive oxygen species (ROS) caused by tissue ischemia, and a direct renal tubular toxic effect by contrast media leading to acute tubular cell necrosis [16]. The production of ROS has been the main pharmacologic target to prevent the development of $\mathrm{Cl}-\mathrm{AKI}$ in various scientific studies, although the protective effects remain inconsistent [17]. Albumin, traditionally viewed as a carrier protein, is in fact also a major natural free radical scavenger in the blood. The cysteine and methionine residues in the albumin structure play a key role in this function [18]. Therefore, low serum albumin level theoretically reflects a state of low free radical clearance capacity, predisposing the body to oxidative injuries.

Hypoalbuminemia has been shown as an independent risk factor for AKI and post-AKI mortality in various clinical scenarios irrespective of contrast utilization. In a meta-analysis performed in 2010 by Wiedermann et al., lower serum albumin served as an independent predictor of AKI [19]. In addition, albumin being one of the negative acute phase reactants, hypoalbuminemia is a reflection of an underlying inflammatory state in the body which is characterized by increased free radical production [20]. Patients who are extremely sick with multiple co-morbidities and organ failure tend to have low albumin because of their profound inflammatory response state and poor nu- trition. Our finding of a significant association between hypoalbuminemia and $\mathrm{Cl}-\mathrm{AKI}$ could also be because of reverse causation bias where the sickest patients are at the highest risk for $\mathrm{Cl}-\mathrm{AKI}$ and they have the lowest serum albumin levels because of the aforementioned reasons. Previous studies have suggested the role of inflammation in pathogenesis of contrast-induced nephropathy using C-reactive protein (CRP) as a marker. In the Athens study, pre-procedural high-sensitivity CRP was validated as one of the predictors of $\mathrm{Cl}-\mathrm{AKI}$ in patients with ACS who had urgent $\mathrm{PCl}$ [21]. Whether hypoalbuminemia is the primary driving factor in causing $\mathrm{Cl}-\mathrm{AKI}$ or it basically proxies other known risk factors of $\mathrm{Cl}-\mathrm{AKI}$ needs to be further investigated by randomized controlled studies adjusting for unknown confounders.

Scientific evidence is scarce on the use of parenteral albumin infusion for prevention of $\mathrm{Cl}-\mathrm{AKI}$. A small retrospective study from South Korea found no difference in incidence of $\mathrm{Cl}-\mathrm{AKI}$ between the parenteral albumin group and the sodium bicarbonate group among patients with liver cirrhosis who underwent contrast CT study [22]. Pre-procedure albumin infusion in comparison to the current standard of care of intravenous saline hydration may be studied as a potentially superior preventive strategy in reducing the incidence of $\mathrm{Cl}-\mathrm{AKI}$ in high risk patients. But cost effectiveness and adverse risks of parenteral albumin infusion will be a significant concern.

Our study has several limitations. First of all, the retrospective nature of the study contributes to selection bias. Patients with no pre-procedural serum albumin level were excluded from this study. Serum albumin level is subject to fluctuations based on hydration status, and due to limited availability of data on retrospective chart review, peri-procedural volume status along with several other factors including urine output and administration of nephrotoxic medications could not be accurately assessed and hence was not incorporated into the regression analysis. Reverse causation bias is inevitable in our study as the sickest patients are at the highest risk for $\mathrm{Cl}$ AKI and they have a low albumin level to begin with. Further randomized controlled studies are required to adjust for unknown confounders to mitigate these biases. Lastly, we did not study the etiology of hypoalbuminemia in our study population and those etiologies might have directly contributed to development of Cl-AKI.

In conclusion, serum albumin $<3.85 \mathrm{gm} / \mathrm{dl}$ is an independent predictor of $\mathrm{Cl}-\mathrm{AKI}$ in patients undergoing coronary angiography. Further prospective clinical studies are necessary to validate serum albumin level as a risk prediction tool for $\mathrm{Cl}-\mathrm{AKI}$ following coronary angiography. 


\section{Conflict of interest}

The authors declare no conflict of interest.

\section{References}

1. Levine GN, Bates ER, Blankenship JC, et al. 2011 ACCF/ AHA/SCAI Guideline for Percutaneous Coronary Intervention: a report of the American College of Cardiology Foundation/American Heart Association Task Force on Practice Guidelines and the Society for Cardiovascular Angiography and Interventions. Circulation 2011; 124: e574-651.

2. Kocayigit I, Yaylaci S, Osken A, et al. Comparison of effects of thrombolytic therapy and primary percutaneous coronary intervention in elderly patients with acute ST-segment elevation myocardial infarction on in-hospital, six-month, and one-year mortality. Arch Med Sci Atheroscler Dis 2019; 4: 82-8.

3. Chen C, Makkiya M, Aronow W, et al. Heightened risk of cardiac events following percutaneous coronary intervention for cocaine-associated myocardial infarction. Arch Med Sci 2020; 16: 66-70.

4. Rear R, Bell RM, Hausenloy DJ. Contrast-induced nephropathy following angiography and cardiac interventions. Heart 2016; 102: 638-48.

5. Seeliger E, Sendeski M, Rihal CS, et al. Contrast-induced kidney injury: mechanisms, risk factors, and prevention. Eur Heart J 2012; 33: 2007-15.

6. Hossain MA, Costanzo E, Cosentino J, et al. Contrast-induced nephropathy: pathophysiology, risk factors, and prevention. Saudi J Kidney Dis Transpl 2018; 29: 1-9.

7. Newhouse JH, Kho D, Rao QA, et al. Frequency of serum creatinine changes in the absence of iodinated contrast material: implications for studies of contrast nephrotoxicity. AJR Am J Roentgenol 2008; 191: 376-82.

8. Lindsay J, Apple S, Pinnow EE, et al. Percutaneous coronary intervention-associated nephropathy foreshadows increased risk of late adverse events in patients with normal baseline serum creatinine. Catheter Cardiovasc Interv 2003; 59: 338-43.

9. Solomon RJ, Mehran R, Natarajan MK, et al. Contrast-induced nephropathy and long-term adverse events cause and effect? Clin J Am Soc Nephrol 2009; 4: 1162-9.

10. Mehran R, Aymong ED, Nikolsky E, et al. A simple risk score for prediction of contrast-induced nephropathy after percutaneous coronary intervention: development and initial validation. J Am Coll Cardiol 2004; 44: 1393-9.

11. Sreenivasan J, Zhuo M, Khan MS, et al. Anemia (hemoglobin $</=13 \mathrm{~g} / \mathrm{dL}$ ) as a risk factor for contrast-induced acute kidney injury following coronary angiography. Am J Cardiol 2018; 122: 961-5.

12. Dai DM, Wang D, Hu D, et al. Difference in hematocrit and plasma albumin levels as an additional biomarker in the diagnosis of infectious disease. Arch Med Sci 2020; 16: 522-30.

13. El Habashy S, Adly A, Kader M, et al. Predictors of future microalbuminuria in children and adolescents with type 1 diabetes mellitus in Egypt. Arch Med Sci Atheroscler Dis 2019; 4: 286-97.

14. Thomas ME, Blaine C, Dawnay A, et al. The definition of acute kidney injury and its use in practice. Kidney Int 2015; 87: 62-73.

15. Murat SN, Kurtul A, Yarlioglues M. Impact of serum albumin levels on contrast-induced acute kidney injury in patients with acute coronary syndromes treated with percutaneous coronary intervention. Angiology 2015; 66: 732-7.
16. Azzalini L, Spagnoli V, Ly HQ. Contrast-induced nephropathy: from pathophysiology to preventive strategies. Can J Cardiol 2016; 32: 247-55.

17. Mehran R, Dangas GD, Weisbord SD. Contrast-associated acute kidney injury. N Engl J Med 2019; 380: 2146-55.

18. Roche M, Rondeau P, Singh NR, et al. The antioxidant properties of serum albumin. FEBS Lett 2008; 582: 1783-7.

19. Wiedermann CJ, Wiedermann W, Joannidis M. Hypoalbuminemia and acute kidney injury: a meta-analysis of observational clinical studies. Intensive Care Med 2010; 36: 1657-65.

20. Kim S, McClave SA, Martindale RG, et al. Hypoalbuminemia and clinical outcomes: what is the mechanism behind the relationship? Am Surg 2017; 83: 1220-7.

21. Lazaros G, Zografos T, Oikonomou E, et al. Usefulness of C-reactive protein as a predictor of contrast-induced nephropathy after percutaneous coronary interventions in patients with acute myocardial infarction and presentation of a new risk score (Athens CIN Score). Am J Cardiol 2016; 118: 1329-33.

22. Choi H, Kim Y, Kim SM, et al. Intravenous albumin for the prevention of contrast-induced nephropathy in patients with liver cirrhosis and chronic kidney disease undergoing contrast-enhanced CT. Kidney Res Clin Pract 2012; 31: 106-11. 